\title{
La gestión educativa: Hacia la optimización de la formación docente en la educación superior en Colombia*
}

\section{Education management: toward optimization of teacher training in higher education in Colombia}

Alejandra Dalila Rico Molano

\section{Resumen}

Este artículo de reflexión tiene como objetivo analizar la gestión educativa como un componente importante en la educación, y más exactamente, el papel fundamental que tiene en los procesos de formación docente en la universidad; la gestión optimiza todos los procesos de la universidad. De este modo, lo primero, es definir qué es gestión, luego, qué es gestión educativa y finalizar con el análisis de ésta en la universidad. Esta distribución permite empezar del concepto general y así lograr comprender lo particular, de lo cual se puede concluir que es necesaria una relación significante entre la gestión y la formación docente.

Palabras Clave: Formación de docentes, gestión educativa, innovación educativa, educación superior, universidad.

\begin{abstract}
The purpose of this reflection article is to analyze education management, as an important component of education, and more exactly, its fundamental role on teacher formation at the university; management optimizes all processes of the university. In this manner, the first thing is to define what management is, then what education management is, and, end with an analysis of such management at the university. This distribution allows to start from the general concept, and in this manner understand the particular, which leads to conclude that it is necessary a significant relationship between management and teacher formation.
\end{abstract}

Keywords: Teacher formation, Education management, Education innovation, higher education University. 


\section{Introducción}

A partir de la década de 1990 en el contexto colombiano, cuando inicia el auge de los cambios en el sistema educativo, partiendo de la reforma constitucional de 1991 y los nuevos procesos de descentralización que atravesaron todos los sectores activos del país, incluyendo la educación, se inicia una nueva forma de percibir el carácter formativo de los docentes. Las instituciones de educación superior incluyen, en sus nuevas reformas, la apertura de formación posgradual que permitirá la profesionalización a un nivel especializado del saber específico que requiera el docente. Este nuevo enfoque de formación docente o desarrollo profesoral toma fuerza a mediados de la década en mención, a tal punto que no solo las universidades cambian sus políticas internas de ofertar más posgrados, sino que se ven en la necesidad de gestionar procesos que brinden formación docente a su cuerpo profesoral. Esta nueva alternativa hace que los docentes estén en la obligación de acceder al sector universitario productivo con una formación especializada tal como especialización, maestría o doctorado, y también, ser capacitados al interior de los recintos universitarios, con el fin de lograr la calidad educativa acorde con los avances de la sociedad contemporánea.

De tal modo, la formación profesoral se ha convertido en una necesidad que requiere ser gestionada desde las directrices institucionales y puesta en marcha de acuerdo con los planes de desarrollo vigentes en las universidades. Esta gestión del desarrollo profesoral ha ocasionado que la mayoría de las universidades tengan convenios con instituciones nacionales e internacionales, con el ánimo de motivar entre sus docentes la formación profesoral continua, brindando estudios de maestría y doctorados, financiados por el mismo recinto, propuesta que establece criterios de desarrollo formativo, profesional y laboral. Desde lo establecido por el marco legal, específicamente la Ley 115 del 94 instituye criterios para la formación de profesores, entre ellos están una alta calidad científica, el desarrollo pedagógico, el apoyo a la investigación y prestación del servicio docente a la comunidad. Además, establece que corresponde a las instituciones de educación superior acreditadas por la ley, con facultades o programas de educación, direccionar el proceso de formación profesional de los educadores en todo el sentido estricto del término. En relación con la formación de los profesores la ley estima:
Artículo 109 d) Preparar educadores a nivel de pregrado y de posgrado para los diferentes niveles $\mathrm{y}$ formas de prestación del servicio educativo. Artículo 110. Mejoramiento profesional. La enseñanza estará a cargo de personas de reconocida idoneidad moral, ética, pedagógica y profesional. El Gobierno Nacional creará las condiciones necesarias para facilitar a los educadores su mejoramiento profesional, con el fin de ofrecer un servicio educativo de calidad. La responsabilidad de dicho mejoramiento será de los propios educadores, de la Nación, de las entidades territoriales y de las instituciones educativas. (Ley 115 de 1994, Ministerio de Educación Nacional, Colombia).

A partir de lo decretado en esta ley, la formación docente se entiende como un proceso permanente y transversal. A raíz de estas nuevas herramientas gestionadas por las instituciones de educación superior, dicha formación se ve inmersa en grandes y complejos desafíos, los cuales se deben abordar desde la misma cultura de la transformación y el desarrollo social. En este contexto las propuestas sobre el tema deben ajustarse a dos grandes realidades: el desarrollo humano y los avances tecnológicos que permean cada vez más los sistemas educativos y la manera de pensar. (Edelstein citada por Rico, A. \& Rodríguez, M. 2011).

Si bien es cierto que la formación docente debe estar acorde con los avances tecnológicos, el pensamiento social y el desarrollo humano, también, se debe tener en cuenta hacia dónde están orientados los procesos de gestión frente a este campo, en consecuencia de las nuevas políticas que articulan aspectos como la calidad, autoevaluación y acreditación de la educación superior, que traen como resultado la necesidad de la permanecía de los docentes y la calidad educativa brindada por la universidad.

Es así como la formación docente está enmarcada dentro de la sociedad de consumo que caracteriza nuestro tiempo. Los cambios acelerados en las instituciones, así como sus políticas, generan una búsqueda constante de competitividad, crecimiento, desarrollo profesional, social y laboral; el concepto de calidad educativa permea cada rincón de la educación, y los docentes como promotores de conocimiento y generación de nuevos profesionales están en la 
obligación de buscar, continuamente, el desarrollo profesional que les permita subir su estatus y manejar de mejor forma los contenidos de su especialidad. Esto hace pensar, como lo afirma Lipovetsky::

En nuestros tiempos se fomenta la ansiedad por la formación, existe ilimitadamente el auge por el consumo educativo, la necesidad por encontrar un estímulos que prolongue la permanencia en la academia hace que cada día existan nueva tendencias y cursos de actualización formativa, la pregunta que desata la intranquila sociedad está latente: ¿Dónde se ve que nuestra sociedad haya dicho adiós al futuro? (Lipovetsky, 2006: 74-76).

Somos individuos que vivimos para el futuro, y esto se ve reflejado en la formación docente: nuevos cursos y dispositivosquesegestionan desdelamismainstitución y que ofrecen no solo un beneficio a la maquinaria escolar, sino al profesorado. Esto es lo que se llama en nuestro sistema educativo: educación de calidad.

\section{La gestión educativa y su correspondencia con la formación docente en la universidad colombiana}

Este aparte está orientado a revisar el papel de la gestión en los procesos de educación superior, ya que hacerle un acercamiento es comprender el proceso, el desarrollo y la proyección que orientan la educación superior. De este modo, se analizará la gestión educativa entendida como el colectivo de recursos, ideas, propuestas y demás dependencias existentes en una institución, con el fin de alcanzar los objetivos propuestos para el desempeño eficaz, eficiente y efectivo de la institución. Al igual, se preocupa por alcanzar los logros en los procesos educativos e institucionales. Se finaliza el artículo hablando de la gestión en la educación superior que contribuye a la optimización de procesos académicos, pedagógicos, investigativos de la institución.

\section{Gestión educativa}

Las nuevas dinámicas sociales y la tendencia hacia la descentralización de los sistemas educativos, genera que los docentes directivos asuman nuevos roles, tengan la capacidad para actuar de manera autónoma y tomar decisiones que le permitan acceder al mundo globalizado de la educación y de la sociedad en general. Todo ello, hace que los actores del ámbito educativo gestionen procesos adecuados y coherentes con la movilidad social que acontece en la sociedad. Quiere decir que se debe asumir responsabilidad para obtener resultados exitosos e innovadores y generar las suficientes capacidades para proyectar, diseñar, analizar y evaluar políticas como proyectos pertinentes al contexto actual. En este marco de ideas, los procesos de gestión de los sistemas de educación, demandan no solo la necesidad de un docente directivo o un gestor de la educación, sino también factores como la planeación, equidad, calidad, manejo de recursos, participación de la comunidad y rendición de cuentas ante esta; todo ello, para generar resultados óptimos y la prestación de mejores servicios.

Bajo esta dimensión, la gestión educativa es entendida como un proceso organizado y orientado a la optimización de procesos y proyectos internos de las instituciones, con el objetivo de perfeccionar los procedimientos pedagógicos, directivos, comunitarios y administrativos que en ella se movilizan. La institución es autónoma de definir sus criterios de gestión más adecuados y encaminados a responder con las necesidades educativas resultantes de la comunidad, más las exigencias legales de corte nacional e internacional. Se considera que la gestión (administración) es un proceso que induce al orden en la institución por tanto:

La administración facilita la estructura y la coordinación de cualquier actividad, con el propósito de lograr identificación y el cumplimiento de los objetivos institucionales (...) la administración en el campo de la educación es un proceso concebido para integrar recursos y coordinar actividades especializadas e interdependientes, en busca del cumplimiento de los objetivos propuestos. (Romero, 1982: 14).

Igualmente, Blanco \& Quesada (s.f.) definen la gestión como un elemento vital para la organización y la calidad de desempeño de cualquier institución, en donde se sitúan dispositivos como el clima organizacional, el liderazgo y los conductos gubernamentales; con estos elementos la gestión 
logra ser un proceso necesario, adecuado y relevante para la movilidad de toda institución. Bajo estos criterios que conceptualizan la gestión y la organización de las instituciones, y considerando que todos los procesos que emergen de ellas deben estar encabezados por personas capaces de dirigir, proponer, liderar y gestionar, de tal manera que se generen resultados que cumplan con los objetivos propuestos institucionalmente, es en este sentido que la gestión facilita la obtención de resultados adecuados y desde luego, la implementación de planes de mejoramiento para la siguiente planeación. Aquí es donde los participantes de los procesos de la institución deben estar al tanto de su dinámica, es por ello, que la gestión de la formación docente es tan relevante como proceso, se hace indispensable para dichas instituciones competentes, generadoras de conocimientos y líderes en procesos educativos de calidad. Al respecto, Salguero (2008) considera que las IES están en la obligación de formar profesionales que se ajusten a las necesidades y exigencias de la sociedad, el Estado y el sistema productivo, de esta manera, uno de los factores que posibilitan esta dinámica, es el rol del docente y su formación profesoral, esta formación debe estar transversalizada por componentes como: las nuevas tecnologías, las políticas en educación, el currículo, la normatividad, entre otros, que permean necesariamente el ámbito universitario y la formación profesoral de los miembros que allí laboran. Al respecto, en un estudio realizado en la Universidad de la Salle, en el programa de Maestría en Docencia, se establece que la gestión educativa es entendida por Betancourt como:

Un proceso en el que se comparten responsabilidades y obligaciones administrativas, decisorias, ejecutorias, de control y vigilancia permanentes, encaminadas a un constante mejoramiento de la educación impartida por el Estado desde sus distintas instituciones públicas y privadas, atendiendo a requerimientos socioeconómicos, políticos, jurídicos, histórico culturales, de distintos entornos, organizados dentro de los parámetros establecidos por el sistema educativo. (Betancourt, 2002: 60).

A saber, la gestión educativa desde esta perspectiva, no solo se observa desde la parte interna sino que debe estar conectada a factores externos, que complementan estos procesos dentro de la institución, bien lo define Betancourt:

\begin{abstract}
La gestión educativa adquiere un sentido más amplio que trasciende las fronteras del sistema educativo, al señalar como responsables del proceso educativo no solamente al Estado y a la institución escolar, sino al delegar responsabilidades a la sociedad y también al conceder alguna importancia a la educación no formal. (Betancourt, 2002:60).
\end{abstract}

Gestionar procesos en el ámbito educativo no solo es fijarse en procedimientos mecánicos y tecnificados, se debe tener claro que la educación tiene como fin el individuo, se educa alguien para lograr un propósito, de esta manera, se planea teniendo en cuenta las necesidades reales de los sujetos que pertenecen a la institución, esta planificación debe tener objetivos que den cuenta de una realidad tangible y tenga presente las ideas de los actores del ámbito educativo. Por otro lado, la toma de decisiones ratifica las acciones a gestionar, en esta toma de decisiones es necesario una participación colectiva y acertada, esto favorece la formulación de estrategias, las cuales determinan la mejor forma de realizar las operaciones propuestas dentro de los objetivos, al igual que es necesario contar con las condiciones externas e internas que rodean la institución. A partir de estos lineamientos es que se gestionan los procesos dentro del ámbito educativo, se empiezan a visibilizar los resultados esperados de acuerdo con los objetivos planteados, para terminar este ciclo con la evaluación del proceso de gestión y encontrar las condiciones más acertadas para retomar de nuevo el proceso, pero esta vez con ajustes que favorezcan los resultados y desde luego el impacto que tiene tanto interno como externo. Optimizar los procesos dentro de la gestión es uno de los aspectos más relevantes, pues allí se determina como un ciclo en donde al finalizar una etapa se inicia otra con modificaciones que generen mejores condiciones dentro de todo proceso, tanto para la institución como para los sujetos.

En este punto, es donde se requiere resaltar que la gestión educativa, además de generar cambios institucionales, exige que genere cambios en los sujetos, en otras palabras, que las estrategias de planificación les otorguen valor como gestores de 
cambio, tomando la definición de Cassasus (1999) quien considera que la gestión es la fusión de los procesos de planeación y administración, ya que aproximadamente unos 20 años atrás estos dos términos eran los que imperaban, pero no en el campo educativo sino en el administrativo o en el económico, empieza a ser relevante a partir de las reformas de la década del 90, en donde el ámbito educativo se ve permeado por las ciencias administrativas y las económicas, estos dos sectores comienzan a discutir en torno a lo que es necesario hacer y lo que se debe desechar en el campo educativo, lo cual trajo consigo el término de gestión. Sin embargo, la idea fundante de añadir el termino gestión a la educación, era precisamente, como se mencionó, generar cambios sustanciales tanto a nivel institucional como en los actores de la misma, pero al parecer, la formulación de estrategias en gestión de la educación, dieron otras matices, en el sentido administrativo, se entendió que únicamente se manejaban insumos y recursos, se vio la educación afectada por el aislamiento de los sujetos frente a los procesos de gestión, bien lo dice Cassasus: "Habría que afirmar, al menos, que lo que resulta de la actividad educativa no es un producto cualquiera. La "materia prima" sobre la cual se trabaja en educación son los alumnos y por lo tanto no se trata de una materia trivial" (Cassasus, 2000: 18), bajo este precepto la gestión en la educación no genera grandes cambios.

Por otra parte, la educación desde hace unas décadas se ve altamente permeada por la economía, y desde esta perspectiva, la educación se refleja en niveles y resultados de impacto, pérdida, ganancias y mercado. Se ratifica esta condición afirmado:

En la última década, los conceptos que dominan la reflexión de la política educativa tienen su raíz en la economía. Conceptos tales como la eficiencia, la eficacia, la evaluación, productividad, competitividad, incentivos (y por cierto la gestión misma) han copado la literatura y el discurso de la política educativa. (Cassasus, 2000: 8).

En este punto, se puede destacar que la gestión en educación esta permeada por factores económicos. Esta entrada de la economía al ámbito educativo, es a raíz de dos razones: la primera, transformaciones socio-económicas que generan nuevas alternativas y enfoques dentro del campo escolar; la segunda, inmersión de la sociedad de consumo, que bajo criterios estrictamente neoliberales, ha ocasionado que la educación como bien público sea controlado por la dinámica del mercado. Convirtiéndolo así en un bien que fácilmente se puede comprar y vender, es decir, en un bien de intercambio financiero. En este sentido, la gestión educativa debe estar bajo la tutoría de los cambios y reglas del sistema económico, bien lo afirma la anterior cita, la eficiencia, eficacia, evaluación, productividad y competitividad son los parámetros a seguir cuando se pone en marcha un plan de desarrollo a nivel institucional o un proyecto educativo, deben estar enfocados sobre objetivos que arrojen resultados tangibles, debe gestionarse bajo tendencias administrativas, organizadas y viables para el mercado de la educación.

Esto ocasiona que la gestión en el campo educativo se convierta en la admiración de recursos físicos, $\mathrm{y}$ no se tiene en cuenta que gestionar en torno a criterios educativos, es un proceso dinamico y que requiere compromiso, principalmente, porque se trabaja con sujetos, y por ello, se requiere de la participación y del compromiso de todos los actores que intervienen, pues, en la medida que exista una seria intervencion, la comunidad se verá inmersa en todo proceso llevado a cabo. Al gestionar educación, se procura el cambio y el beneficio de la institución como de las personas que en ella se encuentran, la gestión de procesos de formación docente es un claro ejemplo de este asunto, si existen criterios claros sobre la formación de profesores, ésta se direccionará hacia la transformación de la educación y la calidad de la institución en cuanto a formación de personas. La formación docente es uno de los retos que tienen las instituciones de educación superior el cual debe ser dirigido teniendo en cuenta, las necesidades que demanda la población a quien va encaminada la oferta educativa, es así como las instituciones de educación superior pueden destinar recursos para una formación coherente con el contexto y con las políticas instauradas de los profesores que allí laboran.

Miñana (1999) afirma que dentro de la gestión educativa lo más importante es generar y apoyar procesos de cambio, en donde los actores de estos procesos sean los que tomen en sus manos la decisión de transformar desde los mismos actos de 
gestión. Significa que al gestionar cualquier proceso dentro de la institución se requiere una orientación para planificar adecuadamente y obtener resultados exitosos, orientación enfocada a fortalecer cada circunstancia, en donde se asuma la autonomía institucional dirigida al cambio y enriquecimiento de todos los ámbitos educativos, con el fin de responder a las necesidades de la población. Igualmente, la gestión está encaminada hacia objetivos y metas definidos, comprobables y pertinentes al contexto, teniendo como objetivo claro mejorar la calidad del servicio educativo prestado a la comunidad y lograr promover el cambio no solo en entes particulares de la institución sino en la totalidad de sus miembros, para aportar valor agregado en conocimientos e innovación tanto en las aulas como fuera de ellas.

De tal forma se considera que gestionar, no solo es conducir a buenos términos una acción dentro de la institución, entiende que la acción:

Atraviesa a toda la institución, en sus relaciones inmediatas, en la coordinación interna, en las maneras de establecer o de frustrar lazos de trabajo, comunidades de trabajo, en la selección de determinados medios, en el conjunto de opciones que se adoptan a la hora de interactuar con otras instituciones. No es solo conducción o dirección, aunque las implique. Es coordinación de procesos de trabajo en el marco de una organización, donde se dan roles y tareas diferenciadas, que en principio pueden ser articuladas generando niveles de gestión. (Huergo, s.f: 3).

Se comprende que en el acto de gestionar no solo participa un grupo exclusivo de personas o como se llamaría "expertos", este es un ejercicio, bien lo dice el autor, en el que se requiere asignar roles y tareas, el primer momento es generar coordinación en el proceso, frente a este asunto de trabajar con los demás, considera Bauman : "Todos vivimos en compañía de otras personas e interactuamos unos con otros" (Bauman, 2007: 17), la distribución de roles y de funciones dentro de la institución depara trabajo en conjunto, la gestión es una acción destinada a organizarse en colectividad, implicando que ésta se convierte en un acto político, además de direccionar, se entrelazan otras acciones como, decidir, dirigir, participar, proponer y actuar, lo político es por naturaleza una práctica comunal, holística y organizada, así es la gestión, de la unificación de criterios depende el cumplimiento de las metas propuestas. Cassasus (1999) sigue esta misma idea, considerando que la gestión es un acto colectivo y que necesariamente debe estar relacionada con el contexto y con la diversidad que se desprende de él, afirmando:

\begin{abstract}
La gestión no está solamente relacionada con el interior de la organización, sino también con el entorno. De hecho, hoy en la gestión la presencia del entorno es cada vez mayor. Todas las organizaciones y particularmente el sistema educativo, son entidades con múltiples puntos de contacto con el entorno y, por lo tanto, con una preocupación especial hacia él. La multiplicidad de la relación interna/externa y los parámetros de la gestión sugieren que el sujeto responsable de la gestión, no tiene que ser necesariamente pensado como un sujeto individual, sino que éste puede ser un sujeto colectivo o un sujeto participativo. (Cassasus, 1999:32).
\end{abstract}

De acuerdo con lo anterior, la gestión educativa está orientada a permitir la integración de todos los actores de la institución, desde una perspectiva participativa y encaminada hacia la toma de decisiones óptimas y direccionadas hacia propósitos que aporten al mejoramiento de la educación. Valderrama (s.f.) considera que cuando se hace referencia a la gestión es hablar de la interacción de personas, en donde se desarrollan acciones en beneficio de estas mismas, quiere decir que la institución es una construcción de subjetividades que interactúan para lograr la configuración de una mejor educación y formación.

La gestión educativa se consolida, bajo dispositivos de cambio, integración, participación, dirección, organización y una evaluación de retroalimentación permanente, estos se desarrollan en la medida que se requiera mejorar o llevar a cabo algo dentro del contexto educativo, de modo que deben existir necesidades dentro del entorno que demanden atención, una planeación y ser gestionadas en plazos razonables y encaminadas hacia metas precisas y con miras hacia el desarrollo tanto de la institución como de las personas que allí se desempeñan. Por esta razón, las instituciones, en este caso de educación superior, no solo deben gestionar procesos coherentes con un 
contexto y la realidad social, educativa y política que se devela en la sociedad actual colombiana, sino que se ven en frente de dispositivos cambiantes y nuevos desafíos, que traen consigno nuevas formas de organización y dirección de todo proceso educativo, esto exige que la gestión educativa sirva para orientar el trabajo y las acciones que allí circulan, teniendo presente la dinámica social, que se dirija siempre al objetivo primario de la gestión: el mejoramiento y el avance hacia la calidad de la educación.

Afirma Cassasus: "La práctica de la gestión hoy va mucho más allá de la mera ejecución de instrucciones. Las personas que tienen responsabilidades de conducción, tienen que planificar y ejecutar el plan" (Cassasus, 2000: 6). Es así como la gestión transforma un entorno educativo y permite establecer parámetros de dirección y ejecución de proyectos relacionados con la educación y la formación, pero, no se debe quedar en dar instrucciones a las sujetos o crear roles de trabajo, debe traspasar esos límites y poder renovar el ámbito educativo, para ello, se ha dejado claro en líneas anteriores, que la integración y configuración de trabajo cooperativo es lo que hace una gestión encaminada hacia la obtención de resultados pertinentes y dirigidos hacia el mejoramiento de la calidad educativa. En últimas, la gestión educativa que requiere especial atención y estudio, no se trata de adecuar los conceptos de la administración o hablar en términos exclusivos de economía y finanzas, de lo que se trata es de lograr relacionar este tipo de concepciones con la formación de sujetos, que, en esencia, es la razón de ser de una educación entendida como la disciplina de formar y construir conocimientos. Así que, se está gestionado con subjetividades y multiplicidad de criterios que hacen de la educación un campo activo y generador de posturas críticas frente a las nuevas dinámicas sociales que emergen actualmente y que deben ser pieza de reflexión en el momento de proponer y emprender acciones de gestión.

\section{Lagestión educativa en lasinstituciones de educación superior en Colombia}

Uno de los aspectos que en la última década ha trastocado y generado serios cuestionamientos en la educación superior ha sido la calidad que brindan las instituciones en cuanto a educación, este tema que produce malestar y preocupación en el gremio, hace que las universidades direccionen sus procesos de gestión por caminos que los conduzca a la calidad total del servicio que prestan. La gestión en este punto se sitúa como la "carta de navegación" que tiene las instituciones paraobtenerlacalidady elreconocimiento en cuanto a formación de sujetos se refiriere. Cassasus considera que el tema de la calidad ha impregnado considerablemente el campo educativo, y manifiesta:

Con la introducción del tema de la calidad en la educación, surgen dos hechos de importancia: por una parte se reconoce la existencia de un "usuario" más allá de las necesidades del sistema, y por otra parte, se genera la preocupación por el resultado del proceso educativo. Es decir, un proceso en el cual se reconoce el derecho de los usuarios del sistema educativo, a exigir un servicio de calidad de acuerdo a sus necesidades. (Cassasus, 2000:11).

Es preciso aclarar que la gestión alude a procesos de calidad, y entiéndase por calidad todo aquello que es beneficioso para los sujetos, es un concepto asociado con el descubrimiento de necesidades en un determinado contexto y la satisfacción tanto particular como colectiva. Se hace referencia a la calidad en aspectos como el desarrollo integral de los sujetos, así como el desarrollo intelectual y social, además, no solo se habla de calidad desde lo cuantitativo, también se debe hablar de calidad (y más en el campo de lo educativo) en términos cualitativos, relacionado con las subjetividades que se desenvuelven en el medio. La calidad es entonces, lo que mantiene la relación estrecha con la satisfacción de recibir y brindar un servicio (educativo) excelente y organizado.

Es entonces cuando emerge la relación educación gestión - calidad, en donde todo proceso educativo debe ser planeado, gestionado y evaluado con un fin último que es la calidad de la educación. La gestión como propuesta para organizar y ayudar a que las instituciones alcancen la calidad esperada en los servicios educativos que brindan a la comunidad, se convierte también en un pilar de acción dentro de los planes de desarrollo de las mismas, a decir verdad este aspecto permea todos los procesos que se desarrollan 
al interior y exterior de la institución, la gestión educativa en los recintos de educación superior es el pretexto para alcanzar la transformación y generar un impacto significativo en la comunidad. De esta forma, la gestión dentro de la institución de educación superior es entendida como el acopio de varias fases (diseño, planeación, ejecución y evaluación) que son el nuevo rumbo o cultura organizacional que caracteriza a la institución, esto significa que frente a este naciente panorama e implementando un nuevo sistema de gestión, también se inicia con una nueva percepción del contexto y posibles respuestas para generar cambios tanto externos como internos.

La gestión educativa en este ámbito contribuye a la optimización de procesos académicos, pedagógicos, investigativos, administrativos y financieros, al igual que abre nuevos espacios de interacción con otros contextos educativos que pueden enriquecer los proceso internos, de esta manera, es como un buen proceso de gestión promueve la formación de los estudiantes, docentes y en general, de la comunidad educativa, es posible que se genere una formación integral y se alcance el reconocimiento social, en la medida que lo gestionado en la universidad traspase la frontera interna y permee el contexto socio-cultural, materializándose en formar más que profesionales, personas de bien y gestoras de cambio social, que logren identificar y ofrecer soluciones coherentes a los retos que día a día emergen de la sociedad actual. Es en este punto, donde la gestión educativa organizada en la universidad, se adhiere al contexto global logrando obtener reconocimiento, formación, desarrollo e innovación.

La gestión es una estrategia que las instituciones, implementan para administrar de manera eficiente los recursos que poseen, para así alcanzar las metas propuestas, en la medida que son una organización. Aristimuño, Guaita \& Monroy (s.f.) consideran "La forma como se hace gestión en las instituciones de Educación Superior, incide en su éxito o el fracaso" (p. 3). Lo que lleva a suponer que la gestión es exitosa en la medida que las universidades diseñen y lleven a cabo políticas de inversión y desarrollo orientadas al progreso y aporten al cumplimento de los objetivos, garantizando la innovación tecnológica, el desarrollo de la investigación y la formación integral y coherente con la dinámica social actual. Con esto, se quiere decir que las universidades ubican en la gestión educativa las herramientas formativas, administrativas $y$ gerenciales para acondicionar las situaciones actuales a un futuro próximo. Es en este sentido, que la gestión permite organizar procesos como la formación docente, ya que este asunto no solo articula procesos académicos de la universidad, sino fortalece el campo investigativo, por este motivo, gestionarla garantiza la innovación y el desarrollo investigativo tanto del talento docente como de la misma institución.

De acuerdo con los planteamientos anteriores la gestión en las instituciones de educación superior está diseñada para organizar la institución y orientarla al cumplimiento de las metas propuestas, como lo afirman Correa, Álvarez y Correa:

\begin{abstract}
La gestión educativa se convierte en una disciplina necesaria para ejercer la dirección y el liderazgo integral en las organizaciones educativas y para lograr el cumplimiento de su función esencial: la formación integral de la persona y del ciudadano, de manera que logre insertarse creativa y productivamente en el mundo laboral. La gestión educativa busca desarrollar un mayor liderazgo en los directivos, con el fin de que ejerzan una autoridad más horizontal, promuevan mayor participación en la toma de decisiones, desarrollen nuevas competencias en los actores educativos, nuevas formas de interacción entre sus miembros y entre la organización y otras organizaciones. (Correa, Álvarez y Correa, s.f: 6).
\end{abstract}

Cabe señalar que la gestión educativa no está orientada únicamente a la planeación de ampliación de infraestructura o a la adquisición de recursos físicos y tecnológicos, es de aclarar que sí es una de las necesidades que debe estar dentro de la gestión, pero no la única ni la más importante. La prioridad debería ser siempre el talento humano con que cuenta la institución, es este factor que debe primar cuando se trata de direccionar, planear, ejecutar y evaluar, en el sentido que el talento humano es el motor primario de la gestión y es lo que tiene con vida a cualquier institución. De tal modo, que al gestionar es importante pensar desde los sujetos que están dentro de este proceso y desde luego brindar soluciones viables para su progreso y desarrollo de competencias integrales. 
Bien se afirmó al inicio de este apartado que las instituciones de educación superior apuntan a gestionar la calidad de la educación que ofrecen a la comunidad, sabiendo que es un proceso complejo y que lleva un buen tiempo alcanzar la calidad del bien que brindan, pues bajo estos parámetros, alcanzar la calidad es tarea de todos los actores del entorno educativo, y es bien sabido que con las nuevas dinámicas sociales y la agilidad con que se transforman los entornos, siempre habrá algo que se considere un reto para alcanzar la calidad, pero en la medida que progrese la sociedad, avance la tecnología y el sujeto moderno tenga más necesidades formativas y profesionales, la universidad se ve en la obligación de ser flexible y organizar permanentemente el objetivo de alcanzar la calidad educativa por medio de procesos de gestión eficaces, eficientes y efectivos. Frente a esta concepción, Botero insiste en que la gestión es el: "Servicio que prestan las personas dentro de las organizaciones" (Botero, 2009: 1), pues si, a pesar de los avances tecnológicos en donde las máquinas y la robótica, está quitándole funciones al ser humano, estos aparatos de "alta definición" no es posible que puedan dirigir una organización creada por humanos, sigue el autor: "Pero en el caso de la gestión educativa, el peso de las competencias humanas es el más representativo" (p. 1). Esto es claro, la gestión es diseñada por humanos para el bienestar de humanos.

Una de las competencias humanas más significativas que tiene un recinto de educación superior es la formación docente, pues, de ella depende el sentido formativo que se imparte en la universidad $\mathrm{y}$ el reconocimiento que adquiere manteniendo actualizados a los docentes, no solo como trasmisores de conocimientos, sino como investigadores y en constate formación con el fin de hacer de esta trasmisión una transformación de conocimiento, tanto en las aulas de clase como fuera de ellas. Entonces, la formación docente es un criterio de gestión diseñada para generar innovación, calidad en la educación y desde luego aspectos que pueden determinar el cambio de pensamiento dentro y fuera de las aulas, es el docente el sujeto que a partir de $\mathrm{su}$ formación logra transformar el pensamiento de los estudiantes y es la universidad el motor de gestión que puede generar este nuevo tipo de pensamiento, fomentando la formación docente desde su misma filosofía y política institucional.
Ahora bien, a esta nueva visión que se abre con el tema de la gestión y la calidad, Botero (2009) considera que la universidad del nuevo siglo, se enfrenta no solo a cambiar de paradigmas sino a visibilizarse desde dentro hacia afuera y viceversa, y abrir las puertas a nuevas disciplinas, como la económica, administrativa, financiera, entre otras, que pueden aportar a una calidad de la educación, que sin el ánimo de transgredir en el objetivo primario de la educación, pueden aportar a este campo de forma óptima. Botero insiste en dejar claro que la educación superior debe estar al ritmo de la dinámica de la aldea global y considera que hay cinco tendencias las cuales se presentan en la universidad del siglo XXI: autonomía, democracia, calidad, formación integral y el surgimiento de la universidad virtual. Es claro que pueden existir otras tendencias, sin embargo, bajo los parámetros de esta investigación, éstas son claves para entender los procesos de gestión de la formación profesoral en la educación superior. En la tabla 1 se explican las cinco tendencias propuestas por Botero (2009). ver sig pág.

De manera que la gestión educativa constituye el centro de operaciones de la institución, es por donde se abre paso a la transformación y la implementación de proyectos con miras al desarrollo institucional, investigativo y social, por estas razones es que al pensar en institución se debe tener en cuenta los procesos de gestión, pues de ellos depende en gran medida el éxito o fracaso, el impacto o rechazo que tiene la institución entre la sociedad. Ahora bien, se habla de gestión en un marco general institucional, atendiendo a los criterios que la definen como un pilar de acción y construcción de la universidad, pero es importante hablar de la gestión de los actores del proceso educativo y formativo, el docente, ¿cuál es ese rol que desempeña este sujeto frente a los procesos de gestión tanto de la institución como de su propio oficio de maestro y promotor de conocimientos? Este sujeto se convierte en agente transformador y generador de conocimiento en la medida que se relacione con cada uno de los procesos organizados en la universidad, se integre a un todo y desempeñe el papel de precursor y guía de lo que acontece en la universidad. De esta manera, como en muchos procesos que se gestionan en un institución se debe pensar en el docente y en su formación, pues desde esta figura es que hay 
Tabla. 1. Tendencias de la gestión educativa. Fuente: Elaboración propia a partir del artículo de Botero.

\begin{tabular}{|c|c|}
\hline Tendencia & Explicación \\
\hline Autonomía & $\begin{array}{l}\text { Entiéndase como el criterio de encontrar soluciones propias que beneficien a la comunidad } \\
\text { educativa en general y al entorno social, la autonomía universitaria busca no solo mirar } \\
\text { hacia dentro sino también se preocupa por atender las necesidades de la comunidad y } \\
\text { buscar en qué medida puede aportar para el desarrollo social, desde la formación de } \\
\text { personas, la investigación y la resolución de problemas propios de la sociedad. } \\
\text { Por otra parte una gestión autónoma trae consigo que la institución cuente con la } \\
\text { posibilidad de tomar decisiones, realizar proyectos propios, coherentes con las necesidades } \\
\text { de los estudiantes y docentes, orientados al mejoramiento del desarrollo de la comunidad. } \\
\text { Afirma Botero (2009): } \\
\text { Ejercer autonomia no significa caos, por el contrario, requiere articular las dimensiones } \\
\text { de la gestión educativa enfatizando en diferentes aspectos de los procesos educativos, de } \\
\text { forma tal que puedan integrarse a teorías que aborden las siguientes dimensiones: } \\
\text { pedagógica, administrativa, social-comunitaria y politica-educativa. (p. } 4 \text { ) } \\
\text { De esta manera la autonomía se logra en la medida que exista la suficiente capacidad para } \\
\text { decidir y actuar, con el objeto de logra bienestar para los sujetos de la institución. Sin } \\
\text { embargo, para las instituciones de educación superior lograr ser autónomas no es tarea } \\
\text { fácil, puesto que deben contar con lo estipulado por los entes gubernamentales, que en } \\
\text { cierta medida quitan autonomía a las universidades, y estas terminan actuando como lo } \\
\text { indican tanto organizaciones internacionales como nacionales. Pero la universidad debe ser } \\
\text { firme en sus decisiones y lograr gestionar proyectos sobresalientes y beneficios para la } \\
\text { sociedad, así el gobierno se dará cuenta que puede lograr aportar pero sin quitarle poder a } \\
\text { la institución. }\end{array}$ \\
\hline Democracia & $\begin{array}{l}\text { Bien lo decía el filósofo griego Aristóteles, la democracia surgió de la idea de igualdad entre } \\
\text { los hombre en cualquier contexto, bajo esta premisa la democracia en la gestión se relaciona } \\
\text { con la inclusión de todos en todo, con la participación colectiva y la toma de decisiones en } \\
\text { común en lo que respecta a la planeación, administración y aplicación de las políticas y } \\
\text { normatividad en educación, pues la educación desde sus orígenes es un bien público, a } \\
\text { pesar de que el neoliberalismo lo vea como un servicio de mercado, las universidades } \\
\text { deben perseguir este fin de la educación. } \\
\text { La democracia en la educación se consagra como el criterio de igualdad, todos están en el } \\
\text { derecho de recibir los beneficios que la universidades ofrece, tanto estudiantes, docentes y } \\
\text { administrativos, la institución debe ser capaz de promover una formación colectiva e } \\
\text { integral que incluya a toda la comunidad educativa y se procure una política de } \\
\text { participación. } \\
\text { De igual forma, la institución está en el deber de impulsar proyectos que acentúen la } \\
\text { integración de los estudiantes en asuntos de la sociedad, es decir crear espacios que } \\
\text { generen el diálogo entre los estudiantes y los actores sociales. Allí es donde se evidencia el } \\
\text { sentido democrático de la gestión educativa. } \\
\text { Finalmente, la sociedad actual agencia dispositivos de desigualdad, marginación y falta de } \\
\text { oportunidades para el ingreso de muchos jóvenes a la educación superior, en Colombia, a } \\
\text { raíz del neoliberalismo, el joven que tiene los recursos puede ingresar, y los que quedan por } \\
\text { fuera deben seguir buscando la oportunidad. Este tipo de conflictos sociales, que se sabe } \\
\text { que cada día se acentúan más, son los que las universidades deben atacar, gestionando } \\
\text { proyectos de inclusión y acercamiento a poblaciones distantes a la educación superior. }\end{array}$ \\
\hline Calidad & $\begin{array}{l}\text { En apartados anteriores se hizo referencia a este concepto, para ampliarlo, aquíse hace un } \\
\text { breve análisis frente a la calidad de educación. Botero (2009) considera que las grandes } \\
\text { transformaciones y las exigencias que genera la sociedad actual, hace que las } \\
\text { universidades demanden formación y talento humano de calidad, sello que puede ser de } \\
\text { reconocimiento internacional. Ofrecer formación de calidad indica una gestión precisa y } \\
\text { acorde con la dinámica social y los avances de la ciencia y la tecnología, es por ello que la } \\
\text { calidad en el campo de la educación no solo responde a un reconocimiento social o una } \\
\text { moderna infraestructura, responde en gran parte al tipo de formación que brinda la } \\
\text { institución, de ello depende la calidad de talento humano que opere en la sociedad. } \\
\text { Sin embargo, para muchas universidades la calidad es un reto, pues se entiende que para } \\
\text { brindar una formación de calidad es preciso también asegurar la formación de los docentes } \\
\text { desde la misma institución, de esta manera gestionar proyectos en pro de la formación } \\
\text { docente es aún una falencia para algunas universidades, no basta con proponer un perfil } \\
\text { docente, es necesario invertir en formación para los profesores, así se puede lograr una } \\
\text { educación dirija a la calidad. En la proyección y gestión de la formación docente depende } \\
\text { en parte el reconocimiento del talento humano que promociona la universidad. }\end{array}$ \\
\hline
\end{tabular}


que planear y proyectar una gestión vista desde criterios socio - políticos y social - culturales, pero también ahondando en el ser y pensar del docente, en criterios investigativos, pedagógicos y formativos.

Bajo esta noción de ver la gestión educativa, y más aún visibilizarla desde la postura del docente, es necesario considerar que en la mayoría de las instituciones de educación superior, se hace referencia a una gestión por procesos, bien se ha dicho a lo largo de este artículo que la gestión es un proceso que busca la calidad de los servicios que ofrece la institución. De manera que una gestión por procesos no solo está dirigida por un grupo de expertos sino que es la integración de todos los actores de la organización, es decir, un proceso se considera como la participación de todos en cada acción que gestione la institución. Correa, Correa \& Álvarez (s.f.) afirman que "la clave de la calidad en una organización está dada por el mejoramiento continuo de los procesos" (p. 19), esto indica que la institución debe organizar procesos adecuados y pertinentes que conlleven al mejoramiento constante de la educación, continúan los autores afirmando:

La gestión por procesos se convierte en una herramienta para direccionar y gestionar los procesos educativos en función de las necesidades, expectativas e intereses de los estudiantes y de la comunidad educativa. Adicionalmente, la gestión por procesos articula temas como calidad, productividad, eficiencia y eficacia; e introduce un elemento más: cómo agregar valor al proceso educativo, lo que implica pensar en cómo innovar en los procesos de enseñanza-aprendizaje. (Correa, Correa y Álvarez: 19).

No obstante, la gestión dirigida por procesos es más organizada y enfocada a la integración de todos los miembros de la comunidad educativa, además, esta orientación comprende no solo temas como la productividad, eficiencia y eficacia, típicos de la gestión, también implica que la institución reflexione sobre el mejoramiento de procesos formativos, de aprendizaje y enseñanza, los cuales se consideran como los que definen el ser y hacer de una institución educativa. Como se planteó líneas más atrás, es necesario ver la gestión desde el quehacer del docente, verlo como un sujeto que transforma y dinamiza los procesos no solo en el aula de clase sino a nivel institucional. Al respecto Correa, Correa \& Álvarez (s.f.) consideran que un gestor educativo es quien genera cambio y está en la capacidad de desarrollar en la institución procesos de innovación y así mismo poder entender las necesidades y las problemáticas que agencia el contexto y la comunidad en general. ¿Quién más idóneo para entender el contexto y aportar conocimiento innovador en la universidad que el docente? Es él quien hace que el estudiante interactúe con el saber y la práctica, es quien sitúa lo aprendido en el aula y forma sujetos sentados en la realidad y que se acogen a las dinámicas de la sociedad.

En otro orden de ideas, es necesario que los docentes se integren a los procesos institucionales y exploren más allá del aula. Cabeza (2004) insiste que la actividad docente involucra aspectos como, la objetividad de la enseñanza, desarrollo pedagógico, formación y actualización permanente, criterios de innovación y aplicación de las nuevas tecnologías, disposición para el diálogo y confrontación de ideas entre pares y estudiantes, finalmente, capacidad para evaluar su labor y los actos cognitivos y formativos de los estudiantes. Quiere decir que el docente es quien genera cambios y permite crear procesos para ser gestionados y que se dirijan al mejoramiento de la calidad de la educación, Correa, Correa \& Álvarez (s.f.), estiman que hoy en día la labor del docente debe ir de acuerdo con los avances y el cambio social: "Es un reto para los docentes en el proceso educativo de sus alumnos y es buscar nuevas y mejores estrategias que permitan seducir y motivar al estudiante para que logre aprendizajes que sean significativos para su vida" (p. 38). Esto indica que el docente debe ser un sujeto preparado y actualizado, él mismo esta auto-gestionando su quehacer y saber, debe tener una mirada holística del contexto, ser mediador, incentivar la investigación y lograr ser un docente investigador de su propio accionar. "El docente es un sujeto social que ejerce la profesión de enseñar y gestionar aprendizajes." (Correa, Correa \& Álvarez, s.f: 39), queda por decir aquí, que gestionar implicar actuar y transformar.

En resumidas cuentas, el docente es quien determina ser un gestor educativo y está en la capacidad de organizar, proponer, decidir, liderar, coordinar y retroalimentar, todo con el fin de reflejar desde la 
gestión procesos óptimos y facilitadores de soluciones a la necesidad que emana la comunidad educativa inmersa en la institución de educación superior:

Se piensa en un gestor educativo que provoca e incita a la acción formadora y por tanto transformadora; que es testimonio de moralidad y compromiso; que une permanentemente a los actores educativos alrededor de la misión y de los objetivos institucionales; que imprime direccionalidad y sentido a la organización y a su quehacer; que evalúa, que construye en el día a día escenarios de esperanza y de futuro (Correa, Correa \& Álvarez, s.f., 45).

El docente gestor no se debe limitar a términos administrativos, es aquel sujeto que reacciona y lidera de forma concreta y eficiente, de esta manera, es capaz de organizar, repensar su quehacer y fomentar el cambio tanto de la institución como de la sociedad. Sin ir más lejos, el gestor educativo tiene la suficiente capacidad de gestionar y transformar la educación, no solo desde un criterio único administrativo sino como docente gestor debe fomentar el cambio desde la implementación de medios que faciliten la formación docente en la institución, bajo aspectos, como la innovación, internacionalización, calidad en la práctica docente y en la investigación.

\section{Formación docente y gestión}

Hablar de formación docente, en el medio universitario, no es hablar solo de pautas para ejercer una mejor labor o de aspectos academicistas o instrumentales es comprender la importancia y el valor social que tiene la profesión, es mirarla bajo criterios de autonomía, integralidad, investigación, desarrollo social y compromiso. Es entonces cuando la formación docente es la propia construcción de su identidad, es la satisfacción de ejercer y ser competente como profesional de la educación, ella debe ser parte del desarrollo personal y estar en relación con el progreso de la universidad, se debe ver como una práctica humana, en donde se comparte conocimiento y se forman sujetos, en ultimas, es una actividad de interacción y diálogo.
Sin embargo, para que la formación docente sea vista como un pilar de avance y calidad de la universidad, se requiere que los mismos docentes generen condiciones para que se establezca como un campo versátil y dinámico dentro del ámbito universitario y desde luego, bajo parámetros de gestión, para ello es conveniente que el docente como sujeto de cambio reflexione sobre:

a. La fuerte resistencia al cambio.

b. Implementación de las nuevas tecnologías en su quehacer.

c. Aceptación de la innovación educativa.

d. Fomentar una enseñanza más teórica y no solo de oficiosidad.

Estos cuatro aspectos deben ser puntos de reflexión tanto en el desarrollo profesoral como en su quehacer, si se logra que los docentes como sujetos transformadores del pensamiento, de lo social y lo cultural, sean los primeros en ser críticos de su propia formación y práctica, y aumenten la participación en las decisiones y políticas establecidas en la instituciones, con ello es más que seguro que gestionar proyectos en torno a la formación de docentes se genere calidad y beneficios bilaterales. Desde esta mirada, hay que tener en cuenta también la postura tanto de los entes gubernamentales como los institucionales internos, siendo estos los que presentan ante la comunidad una serie de normas y políticas que determinan todos los campos de acción de las universidades, estas políticas normalmente están condicionadas por numerosos factores, como el avance tecnológico, los cambios políticos, las crisis económicas y la intervención de entes internacionales; factores que determinan cómo deben ser los procesos formativos, investigativos y funcionales de toda la universidad, quiere decir que la institución debe apuntar al reconocimiento global, para así tener mejores resultados y una posible educación de calidad. Pero más allá de la intervención externa y la asimilación interna de todos los criterios y las políticas educativas, es necesario que estos entes dirijan sus reflexiones y decisiones a aspectos como:

a. Motivación del profesorado para su mejoramiento en la formación y práctica. 
b. Diseño de políticas de formación y desarrollo profesional coherentes y de calidad.

c. Instauración de modelos pedagógicos pertinentes al contexto y la realidad social del país.

d. Autonomía docente y apoyo institucional.

e. Mejores condiciones laborales y acreditar valor a la labor del docente universitario.

f. Inversión en la investigación no solo de carácter técnico, también de las ciencias humanas y sociales.

g. Una política de calidad de la formación docente, teniendo en cuenta las necesidades e intereses de los docentes.

h. Tener en cuenta que los docentes universitarios requieren formación permanente para estar al tanto de los avances tecnológicos, los cambios sociales y la dinámica de los sujetos que forman.

A propósito Torres (2001) considera que los docentes deben ser reconocidos como profesionales, interlocutores y sujetos del cambio educativo, esto implica dejar de ver la función de estos en la dinámica de "diseño - ejecución", es más bien: "Crear espacios y mecanismos de diálogo, participación y consulta permanentes, ver al docente como individuo y colectivo, tanto en el ámbito educativo como en el de las políticas educativas" (p. 21). De ello se desprende que la formación docente en la universidad debe estar organizada mediante procesos de gestión pertinentes, eficaces y viables para poder solventar las necesidades formativas, investigativas y profesionales de los docentes, quiere decir que la gestión de la formación docente no solo congrega a los directivos sino a los docentes en su colectividad.

Las instituciones de educación superior requieren asumir como estrategia de gestión la formación docente, en la medida que se constituyan proyectos destinados hacia el mejoramiento permanente de la formación docente, la profesionalización y brindar una educación de calidad e integral, así como el propósito de formar profesionales idóneos y capaces de afrontar las problemáticas actuales de la sociedad moderna. De esta manera, la universidad debe tener claro que para lograr una educación con calidad es necesario invertir en la formación de los docentes, se debe consolidar como un reto dentro de los planes de desarrollo de las instituciones, pues brindar formación es tener la posibilidad de dirigirse hacia la calidad, la innovación y altos estándares de investigación. La posibilidad de tener varias opciones de diplomados y cursos financiados por la universidad para la formación integral del docente, hace que esté en constate desarrollo intelectual y profesional, la universidad requiere comprometerse con los docentes y brindar una formación encaminada hacia la mejora continua de los sujetos que forman y la profesionalización de los profesores para que su quehacer sea más innovador, autónomo y crítico, esto quiere decir que debe necesariamente estar altamente comprometida con gestionar formación docente en pro de la docencia y la educación.

Por otro lado, la gestión de procesos de formación docente es necesario en cualquier institución con miras a tener una educación con calidad, y no solo eso, la exigencia del mercado hace que las universidades además de tener excelente infraestructura, requieren que se le otorgue valor a la labor de los docentes, no solo de tipo económico, también, valor a la labor del profesor es garantizar las oportunidades de su profesionalización, formación y desempeño dentro de la universidad, de esta forma, se está ahondado en las necesidades del grupo docente y abarcando las necesidades de la institución y su meta de alcanzar una educación de calidad.

De la misma forma, es necesario elaborar un diagnóstico que permita saber cuáles son las necesidades de la comunidad docente $\mathrm{y}$, desde luego, la que se forma, adicional se requiere hacer convenios con otras universidades tanto nacionales como internacionales, las cuales tengan programas que permitan la formación y desarrollo profesoral, de modo que los docentes puedan verse beneficiados y ampliar su profesionalismo y las competencias 
en su disciplina. Estos convenios para la formación docente, deben contar con programas de maestrías, doctorados y posdoctorados, en donde exista una financiacion real por parte de la institución y continuar formarndose como docente e investigador. Frente a este aspecto, se requiere profundizar, comprendiendo tambien, que la formación docente no es únicamente el escenario donde se obtienen títulos porque sí, es necesario verlo desde perspectivas, como la formativa, investigativa y el mejoramiento del quehacer docente. No basta con otorgar becas de estudio posgradual, es ineludible que la universidad vea también que para ello se requiere destinar un tiempo y que el docente pueda contar con el apoyo de la institución, en el sentido, de reducir la asignación académica y administrativa del docente en formación, otorgarle más horas de dedicación a su formación, significa que la institución debe repensar las políticas del desarrollo profesoral y apoyar al docente en su proceso de formación, el cual es necesario para que la institución pueda adquirir más reconocimiento y alcanzar los estándares de una educación con calidad.

Es preciso recalcar que las instituciones de educación superior han de gestionar formación docente, no solo enfocada a los estudios de maestrías o doctorados, o la participación en espacios académicos, como congresos, simposios, ponencias, entre otros. La institución con el fin de apoyar la formación, la investigación y enriquecer el quehacer del docente, necesita gestionar otros espacios de formación, para contribuir de manera directa al mejoramiento continuo de la calidad de la educación y ayudar a dinamizar los procesos académicos institucionales. Estos espacios se dan con oportunidades, como lo es la publicación de artículos y libros de tipo académico e investigativo resultado de la formación docente constante y dinamizada desde la misma institución, el compromiso con la calidad de la educación es la máxima de la universidad, no solo para adquirir reconocimiento social o brindar un mejor servicio educativo sino poder contar con un talento docente competente, eficiente y eficaz preocupado por su formación docente, coherente con la dinámica de la sociedad actual y desde luego crítico frente a los cambios sociales, económicos, políticos y culturales que afectan la comunidad en formación.
Dentro de este contexto, Cruz, Molina, Herrera, Marentes \& Cubides (1998) consideran:"Para un cambio y una educación de calidad se requiere un maestro renovado" (p. 29), es precisamente lo que encierra la gestión de la formación docente en la universidad, procurar que los docentes sean sujetos que promuevan el cambio social, comprometidos con la educación y la transformación del país. Estos autores presentan unas características que debe tener el docente preocupado por su formación docente y su quehacer, estas son, la preocupación por la formación en valores, producir aprendizaje significativo, educación integral, actualización, comprender el por qué y para qué enseñar y un educador innovador. Estas características logran definir en gran parte el propósito de la gestión de procesos de la formación docente y hacia donde debe ir dirigirá desde la misma universidad, al igual que definen el fin último de este proceso investigativo, que es fijar la formación docente y su gestión desde el ámbito universitario actual.

Al respecto, conviene decir que estas características ejercen un papel importante dentro de la formación docente que debe gestionar la universidad, pues es importante que los docentes reflejen compromiso, interés, integralidad e innovación en su quehacer, y por eso, es que la universidad debe partir de la idea de contar con un talento docente idóneo e interesado por su formación permanente, con sentido crítico frente a los cambios sociales que se presentan constantemente. En últimas, la gestión de la formación docente en la universidad es un proceso que ha de llevarse a cabo teniendo en cuenta las necesidades de la comunidad universitaria y el avance que tenga la institución, pues, como se ha venido insistiendo, la formación docente es un tema muy discutido y que requiere especial atención por parte de los organismos encargados de brindarla, llámese universidad, gobierno, entre otros, porque no es solo sacar al mercado programas de posgrado, es ante todo formar sujetos que están formando a otros, también es disponer de programas coherentes con la realidad social y relacionados con las nuevas tecnologías y la nueva dinámica de la sociedad, en definitiva, la formación docente no consta siempre de obtención de títulos, es ver reflejado en el docente su compromiso, identidad, valor por su profesión y desde luego su capacidad para comunicar conocimientos, es ver un docente 
que transforma y genera pensamiento autónomo frente a los cambios sociales y la investigación.

\section{Conclusiones}

Una primera conclusión que queda sobre la gestión de los procesos de formación docente en las instituciones de educación superior en Colombia, es comprender que ésta es un criterio de suma importancia para la generación de investigación y mejor educación y poder brindarle a los docentes la posibilidad de formación continua, sean programas de posgrado, becas, participación en eventos, publicaciones, investigación, entre otros. Con ello no solo se garantiza una mejor educación, sino también, la profesionalización del cuerpo docente y el reconocimiento de la institución, cabe aclarar que esto es un bucle necesario, el hecho que la institución también se beneficie no es un factor negativo, es una consecuencia de la misma labor de poder gestionar procesos de formación docente, de hecho, este bucle es fundamental y bajo criterios de acreditación siempre estará presente.

Por otra parte, desde las tendencias propuestas por Botero (2009) frente a la gestión en la educación superior, las cuales son, autonomía, democracia, calidad y formación integral, la universidad debe divulgarlas como estrategias que procuren una mejor formación. En cuanto a la autonomía es importante generar conocimiento, investigación y altos estándares de profesionalización, debe ser autónoma y tener capacidad para proyectarse hacia el futuro con el fin de generar cambios en el ámbito de la educación y la misma docencia. Frente a la democracia, la universidad es un espacio donde se construyen constantemente criterios para que se pueda respetar las ideas de las personas, que todo proceso sea compartido y se puedan optimizar las dificultades y brindar mejores soluciones educativas a la comunidad; debe ser una universidad incluyente. Por otra parte, desde la calidad, es evidente que con los actuales parámetros de acreditación institucional, la universidad debe apoyar y gestionar especialmente los procesos de formación, pues este criterio es transversal a todas las instituciones y los docentes son el motor de estas organizaciones.
Finalmente, pensar en gestión de la formación de docentes colombiano es dirigir la mirada hacia el valor que tiene la docencia, es darle sentido a la identidad de este saber, es procurar ir más allá de conseguir un título de maestría o doctorado, no basta con tenerlos, sino darles sentido, y ese sentido se logra en la medida que las instituciones de educación superior tengan políticas que cobijen el esfuerzo del docente por ser mejor cada día y poder propagar mejores y más sólidos saberes en el aula de clase. La institución como ente que procura una mejor educación, debe dejar de burocratizar y mercantilizar la profesión docente y gestionar realmente formación, gestionar conocimiento e investigación. Este país necesita de esos procesos, en donde se evidencie que el docente es quien transforma no solo un contexto, sino es quien transforma mentes y es consciente del verdadero cambio y todo lo refleja desde su formación sólida y consolidada en la búsqueda constante de razones para transformar desde el aula hacia el entorno social.

\section{Referencias bibliográficas}

Aristimuño, M., Guaita, W. \& Monroy, C. (s.f.). Las competencias gerenciales en la gestión de instituciones de educación superior. Disponible en: http://www.ucv. ve/fileadmin/user upload/vrac/documentos/ Curricular Documentos/Evento/Ponencias/ aristimulo y otros.pdf

Betancourt, E. (2002). La gestión educativa ante la violencia intrafamiliar en estudiantes de básica primaria del sector oficial, urbano Melgar (Tol). (Tesis de Maestría). Facultad de Educación. Bogotá: Universidad de la Salle. URI: http://hdl.handle.net/10185/14792

Bauman, Z. \& May, T. (2007). Pensando sociológicamente. Buenos Aires: Nueva Edición.

Blanco, I. \& Quesada, V. (s.f.) La gestión académica: criterio clave de la calidad de gestión de las instituciones de educación superior. Disponible en: http://www.ucv. ve/fileadmin/user upload/vrac/documentos/ 
Curricular Documentos/Evento/ Ponencias_1/Blanco_y_Quesada.pdf

Botero, C. (2009). Cinco tendencias de la gestión educativa. Revista Iberoamericana de Educación, 49, (2), 2 - 11. Disponible en: http://www.rieoei.org/ deloslectores/2811Botero.pdf

Cabeza, M. (2004). Indicadores de gestión en la educación superior como herramienta de la planificación estratégica. Revista venezolana de análisis de coyuntura, 10 (2), 105 - 116. Disponible en: http://redalyc.uaemex.mx/ pdf/364/36410206.pdf

Cassasus, J. (1999). La Gestión: en busca del sujeto. Santiago de Chile: UNESCO. Disponible en: $\quad$ http://es.scribd.com/doc/12667410/ gestionbuscadelsujeto

(2000). Problemas de la gestión educativa en América Latina: o la tensión entre los paradigmas de tipo $A$ y de tipo $B$. Santiago de Chile: UNESCO. Disponible en: http://www.lie.upn.mx/docs/ Especializacion/Gestion/Lec2\%20.pdf

Correa, S., Correa, A. \& Álvarez, A. (s.f.). $L a$ gestión educativa un nuevo paradigma. Disponible en: http://virtual.funlam.edu.co/ repositorio/sites/default/files/6lagestionedu cativaunnuevoparadigma.pdf

Huergo, J. (s.f.). Los procesos de gestión. Disponible en: http://servicios.abc.gov.ar/lainstitucion/ univpedagogica/especializaciones/ seminario/materialesparadescargar/ seminario4/huergo3.pdf

Ley 115 de 1994, Ley General de Educación. Disponible en: http://www.mineducacion. gov.co/1621/articles-85906_archivo_pdf. pdf.

Lipovetsky, G. (2006) Los tiempos Hipermodernos. Barcelona: Anagrama.
Miñana, C. (1999). En un vaivén sin hamaca: la cotidianidad del directivo docente. Bogotá: Universidad Nacional de Colombia. Programa RED.

Rico, A. \&Rodríguez, M. (2011) La Formación Docente Inicial en cuatro décadas a partir de historias de vida de maestros colombianos. Tesis de maestría. Facultad de Educación, Bogotá: Universidad de Salle.

Romero, A. (1982). Administración de la educación: introducción al estudio de la gestión administrativa en las instituciones escolares. Bogotá: Universidad Santo Tomás.

Salguero, L. (2008). Gestión docente y generación de espacios organizacionales en las universidades. Revista de educación Laurus, Vol. 14 (27), 11-32. Venezuela: Universidad Pedagógica Experimental Libertador. Disponible en: http://redalyc. uaemex.mx/src/inicio/ArtPdfRed. isp?iCve $=76111892002$

Torres, R. (2001). La profesión docente en la era de la informática y la lucha contra la pobreza. Séptima reunión del Comité Regional Intergubernamental del Proyecto Principal de Educación en AL y el Caribe. Unesco.

Valderrama, J. (s.f.) La gestión educativa hacia el mejoramiento de la calidad de la educación. Disponible en: http://www.medellin.edu. co/sites/Educativo/Directivos/Medellin/ Biblioteca/Calidad.pdf. 